\title{
Informational Challenges in Early Disaster Response: The Massive Oso/SR530 Landslide 2014 as Case in Point
}

\author{
Hans J Scholl, Stephanie Ballard, Sarah Carnes, Andy Herman, and Neal Parker \\ University of Washington, The Information School \\ \{jscholl, ballard4, sic12, andyh17,nap23\}@uw.edu
}

\begin{abstract}
In disasters, and more so in catastrophes, one of the most daunting problems to professional responders is gaining situational awareness. Unfortunately, truly actionable information (intelligence) is missing in the first days and even weeks of responding. Consequently, incomplete situational awareness brings on a distorted common operating picture leading to suboptimal direction of responses, so more lives are lost, and more damage is inflicted. This study reports on the challenges emergency responders faced in the immediate aftermath of the 2014 massive landslide near Oso, WA. Over a hundred agencies were involved in the response, which presented a huge coordination task for the incident command. This study identifies and describes various challenges in the early response and also discusses recommendations on how to tackle and potentially mitigate the challenges identified in future responses.
\end{abstract}

\section{Introduction}

On March 22, 2014 the most massive landslide (not attributable to volcanic activity) in the history of the United States occurred near Oso, Washington. It killed forty-three people as well as an unknown number of animals. It also destroyed over fifty residential structures and cut off Washington State Route 530 (SR530) at a length of more than one mile.

After the immensity of the incident was understood, President Obama declared it a national disaster. The response and early recovery effort extended for almost three months, and the SR530 only opened to the public in late September, after it had been freed from the mud cover and partly rebuilt in the worst affected area. To this day, the debris field looks like a moon landscape, and the affected local communities recover only slowly.

A total of 119 agencies from all levels of government were involved in the response including the National Guard, the Coast Guard, the State Emergency Management Division, FEMA Region X, Snohomish County, the Department of Natural Resources, EPA, among others as well as dozens of re- lief organizations. More than a thousand individuals participated in the response effort.

Response efforts on this scale are extremely complex undertakings, and they require enormous managerial, operational, and tactical skills on part of the responders. Responders use many traditional and novel technologies to do their jobs. They have to rely on effective information infrastructures to facilitate what is called "situational awareness," which then can be formed into a so-called "common operating picture." In other words, accurate and reliable information is the most important and most scarce resource in early disaster response. A lack of situational awareness leads to issues in the coordination of response units and presents challenges to the incident command system, which is widely (and, for that matter, successfully) used in US disaster response. For this study, first responders from several levels of government were interviewed, among whom were the local responders first on site, members of the Snohomish County Emergency Operating Center, the incident commanders, responders from neighboring municipalities and counties under mutual-aid agreement as well as urban search and rescue teams (USAR), the State Emergency Management Division (EMD), the WA National Guard, and FEMA Region X.

The paper is organized as follows: First, the extant literature on disaster response and situation awareness is reviewed. Then, the study's research questions are outlined, followed by the methodology section. Next, the findings are presented succeeded by a discussion of the insights from the findings. Finally, conclusions are conferred along with directions for future research on the subject.

\section{Literature Review}

The mission-critical role of actionable and integrated information in disaster response has increasingly been emphasized in recent research ([10, 21, 24]). Shared situational awareness leading to a shared common operating picture $[9,10,17,24]$ are considered foundational to agile, disciplined, and effective emergency and disaster response [16]. However, the larger the disaster the more agencies respond, and the 
more complex is the coordination of action [7, 18]. Gaining and maintaining shared situational awareness in "the complex, heterogeneous emergency management structure" is seen as "exceedingly difficult," and "the assumption that shared situational awareness will be easily achieved are doomed to failure" [17, p. 1]. Endsley who laid the foundations to a theory of situation awareness (SA) in the context of combat aviation $[8,9]$ recently recognized an unaddressed and vast research problem surrounding SA, in general, and shared SA, in particular, by pointing at a "myriad of problems" with regard to "data overload, poor integration of information to support comprehension and projection,...poor information representation, and inadequate flow of information..." [10, p. 164]. However, despite the obvious need for addressing these informational problems in SA few studies, if any, were found, which incorporate an information perspective.

The three levels of perception, comprehension, projection in SA [9] are dynamically intertwined implicating level-specific and stakeholder-specific information needs, which require systematic study. Furthermore, the information needs of disaster responders on each SA level lead to specific information behaviors [3]), which may vary individually as well as for larger groups. Extreme event-related information needs and information behaviors in turn entail SA level-specific and responder-specific information flows. These information flows and their potential disruptions also need to be studied in detail. The information perspective provides a sharp lens on disaster-specific information needs, information behaviors, and information flows among and between responders during an unfolding disaster.

The information perspective is a human actorand action-centric perspective. It is concerned with technologies as the potential facilitators of information needs, behaviors, and flows, but not for their own sake. Information and communication technologies (ICTs) have assumed increasingly important roles in supporting and facilitating information behavior and information flows, in general. In disaster management, they have become key elements $[4,18]$ in providing high-quality, mission-critical, and timely and actionable information, fulfilling the rapidly changing ad-hoc information needs of first responders $[18,19,26])$, although they can also be the cause for information overload, work overload, and other stressors [10]. Information behavior and information flows under disaster conditions depend on robust and resourceful information infrastructures, which are embedded in social structures and processes (including formal and informal organizational processes) as their key elements $[6,22,26]$.

\section{Research Questions}

The research questions that guide this exploratory inquiry are targeted at narrowing the recognized gap in the literature with regard to SA along the three central information dimensions, which are (1) responders' specific SA-related information needs, which in turn lead to (2) certain $S A$-related information behaviors of disaster response teams (for example, in terms of seeking, collecting, or verifying information) as well as to (3) vertical and horizontal $S A$-related information flows between and among disaster response teams. This leads to the following three compound research questions, all of which address the whats, hows, and whys, as well as the challenges in each informational dimension:

\section{Research Question \#1 (RQ\#1):}

What are specific $\boldsymbol{S A}$-related challenges for disaster response teams on different levels of involvement, and what are specific challenges to meet the information needs?

\section{Research Question \#2 (RQ\#2):}

What are specific $\boldsymbol{S A - r e l a t e d ~ i n f o r m a t i o n ~ s h a r - ~}$ ing challenges for disaster response teams, and what are specific challenges to responders' information behaviors?

\section{Research Question \#3 (RQ\#3):}

What are other challenges in the context of acquiring and maintaining SA among and between disaster response teams, and what are specific SArelated challenges to responders' information flows?

\section{Method Section}

Instrument and Coding Scheme. Based on the conceptual framework of resilient information infrastructures (RIIs) [24] a semi-structured interview protocol was devised upfront, which covered six topical areas of (1) management and organization, (2) technology, (3) governance, (4) information, (5) information infrastructure, and (6) RIIs/resiliency. A total of thirty-six interview questions and probes were incorporated.

Sample. The sample was purposive [23] and included responders from nine different groups: the (1) local responders, (2) County Emergency Operations Center, (3) urban search and rescue teams, (4) WA State (type-2) response teams, (5) responders from neighboring jurisdictions under mutual aid agreements, (6) State Emergency Management Division, (7) WA State Department of Transportation, (8) WA State National Guard, and (9) Federal Emergency Management Agency (FEMA), region X. A total of 31 individuals were interviewed. 
Data collection: Interviews were conducted in person between September 2014 and March 2015 and lasted between 55 to 261 minutes. One interview was conducted via Skype video conferencing. All interviews were audio taped, transcribed, and coded by at least two coders for analysis. During the interviews also notes were taken and participant interaction was observed and recorded. Moreover, other documents such as after-action reports and press interviews were collected, reviewed, and coded as appropriate.

Data analysis and coding: The initial codebook, which was based on the aforementioned conceptual RII framework, contained six category codes (one for each topical area) and 134 sub-category codes. Additional codes were inductively introduced during data collection, in individual coding sessions, and intercoder sessions [14, 15, 25, 27]. Since a codebook in a hybrid approach of deductive and inductive analyses [12] is designed to be open to extension, it ultimately encompassed 151 sub-category codes in the six main categories.

At least two researchers coded each transcript and document by means of a cloud-based software tool for qualitative and mixed-method data analyses (Dedoose main versions 6 And 7, dedoose.com). The coded data were compared one by one and demonstrated high inter-rater reliability.

The code frequency table revealed the highest numbers of code applications in the areas of "management and organization" $(2,219)$, "technology" (968), and "information" (711). For the purpose of the specific analysis on situational awareness-related information needs, information behaviors, and information flows the code intersection represented by the sub-codes of "situational awareness," "address challenges of information sharing," and "use of information and communication technologies for information sharing" was selected, which produced 650 excerpts.

Excerpts, which were between two and three paragraphs in length, were organized per responder team and conceptually analyzed. Recurring themes and main concepts were identified and named by means of key phrases and keywords. These concepts/context clusters were transferred to the "canvas" of a cloud-based mapping tool (CMAP, version 6.01.01). The concepts/context clusters were inspected and sorted into topical "bins" or "baskets," in which chronological, logical, and other relationships were identified. Relationship links between concepts/context clusters were established whenever evidence from the data supported that link.

Research team and processes. The research team consisted of the principal investigator (PI) and more than forty research assistants (RAs), both for-credit and voluntary. The PI and RAs worked individually and in small teams to transcribe, code, and conceptually/contextually analyze, and map the concepts. The research team met weekly in person or online and communicated via the research project site and the project listserv as well as via individual face-to-face and group meetings. All weekly meetings were streamed and recorded, which kept the whole research team in sync over extended periods of time.

\section{Findings}

5.1 Ad research question \#1 (responders' specific SA-related challenges):

Gaining full situational awareness is acknowledged as one of the most vexing challenges to responders on all levels. This appeared to be no different in the case of the Oso/SR530 landslide response. Responders were in urgent need of acquiring reliable and accurate information on the extent and impact of the landslide. However, several challenges to gaining and maintaining SA were discovered across responder teams, predominantly during the first 24 to 72 hours into the incident. But even after 72 hours several SA-related challenges persisted.

Almost all responder groups mentioned the lack of sharing information in a timely fashion among responder groups during the early phases of the response, which in turn led to incomplete SA and a distorted common operating picture (COP). It appears from the data that shared situational awareness in terms of capturing the basic facts (and with it an initial shared COP) emerged only after 72 hours into the incident, if not even later. Interviewees attributed this delay in arriving at a shared SA to several intertwined factors: (1) in a geography known for frequent mudslides, the term "mudslide" or even "landslide" in early responder communications inadvertently framed a mental model pointing at a far minor incident than the one at hand. It took ground zero responders quite some time to change their mental model; however, also responders at County and EMD levels initially worked under the regular "mudslide" assumption. One responder remembered it with some explicit verbiage,

So initially, it was like, hey, there's a mudslide in Darrington, and it was like, so big $f^{* * * i n g}$ deal, there's mudslides everywhere. No, it's a big one, yeah, OK, big $f^{* * *}$ ing deal, it's a big mudslide. And then it was like, No, it's a really $f^{* * * i n g ~ b i g ~ o n e . ~ O h, ~ m a y b e, ~}$ we, you know, we might end up going to this. So we asked our leadership.

(2) The local responders on the ground had only a partial view onto the site of impact; on the Western 
(Oso) side responders initially even thought that the slide might have come from the Southern hillsides. Their view on the failed slope was blocked, whereas the Eastern (Darrington) side responders had a clear view on the slope, which, however, did not necessarily indicate a huge hillside failure from the angle and distance of observation; as a result, initially responders on neither side understood the extent of the slide and the large area impacted; rather than being separated by debris from each other by about 100 or 200 yards on the blocked and buried SR530 as they thought, they were in fact about a mile apart from each other. (3) County and other helicopters reached the debris field as early as 35 minutes after the landslide had occurred and engaged in search and rescue operations missing to systematically report back to the base about what they saw. However, while the aircrews clearly noticed the extent of the debris field, they were unable to identify the conditions on the ground, which differed substantially on the Western and Eastern edges of the debris field. Said one interviewee,

On Monday < March 24, 2014-insertion by authors> I don't think everybody had a full idea of the scope of the slide yet, where the edges were. I know on Sunday they didn't. It blew me away that we had, I forget how many helicopters in that initial response, and I've heard it at a number of these debriefings "Well, they were too busy doing rescues to tell anybody what was going on."

Another interviewee held,

It was all about information. And an ability to articulate what you saw, and what you thought you understood, as well as be able to articulate the question: "What is really happening." I think that didn't get asked right away. Because people were so focused on what they know, or what they thought they knew. It created a sense of tunnel vision almost for them.

And yet another responder affirmed,

And I didn't fully probably, 100\% understand the enormity of it until I got out there and walked it, and walked from one end to the other in the mud and saw what our people were up against. On day 5 I did that.

Although responders on the ground and in the air separately knew essential elements of information such as (1) affected area (aircrews), (2) characteristics of the debris on the Western side (Oso responders), as opposed to (3) different characteristics of the debris on the Eastern side (Darrington responders), these elements were not immediately integrated to establish shared situational assessment, and subsequently, no shared common operating picture. One interviewee stated,

I don't think we had a central point for all that information to be reported through, and it kind of goes back to that thing we were talking about-information from the field being collected at a single point at the incident command post, being passed up to the EOC. And then information that's coming into the EOC that we pass back down to the incident command post, because it bypassed them.

Another interviewee said, At the beginning, I mean, the estimates that were coming from the Army Corps of Engineers and WSDOT were insane. Insanely low, insanely high, there was nothing you could believe, and so there was always this kind of feeling of "We don't know where we're at. We don't know what we're doing, we don't know what the impact is."

According to interviewees from various teams, communication and information sharing between the local incident command posts, the County EOC, which was not even activated to the highest level, and the fully activated State EOC, particularly on day 1 and 2, but also into the first week of the response were slow and incomplete, which prevented shared situational awareness among response teams and the development of a shared common operating picture and coordinated incident action planning.

Since the slide had remained active for an extended period of time, and the thick debris in many areas was wet and initially proved too dangerous for extended ground operations, search and rescue by air soon became the sole option. This, in turn, kept aircrews from systematically reporting to the ICP and the EOC essential elements of information such as the identified extent of the slide and the boundary of the impacted area.

While on the day of the incident the response was mostly handled by local responders from the Oso Fire Station, the County Fire District 21, the Darrington Fire Station, State Troopers, the Arlington police, and the County Helicopter Rescue Team, the Regional type-3 team took over the incident command on day 2. The type- 3 team was followed by the first of two State type-2 teams beginning on day 6 (Thursday, March 27, 2014). These gradual shifts from lower to higher-level and higher-capability response teams also demonstrate the growing situational awareness regarding the magnitude of the incident. The timing of the shifts shows the enormity of the incident was better understood no sooner than 
Wednesday night, March 26, 2014). However, the handovers between teams also resulted in some friction and loss of situation detail knowledge. As an interviewee said:

The communication after that between the EOC and the IMT and the group out here working and the little satellite places like this fire department and Darrington Fire Department and Arlington Fire Department... Communication between all these entities got a little fuzzy in there. And there's where the email chain got lost.

Another responder added, And towards the end, I think we had a much clearer view of "This is what we're going to need, <and $>$ this <is $>$ what the next...six weeks is going to look like." And we could start seeing that. But on day six, you didn't have that.

In summary, responders understood the enormity of the landslide only gradually, that is, situational awareness was established only slowly. This can be attributed to operating from an incorrect mental model (local mudslide) and the initial lack of systematically collecting and sharing essential elements of information, which hampered the establishment of a shared common operating picture, which in turn complicated the coordination of response efforts. With increasing understanding of the enormity of the incident at hand, higher capability response teams were activated and deployed. However, the handovers from lower-level incident management teams to higher-level teams also led to loss of information detail.

5.2 Ad research question \#2 (information sharing challenges):

Sharing essential elements of information (EEI) among response units regarding the incident at hand, particularly, synchronously and in real time, is key to developing a comprehensive shared SA/COP and a coordinated response. For this to happen the incident management team (IMT) at the incident command post (ICP) along with the cognizant jurisdiction's supporting emergency operations center (EOC) need to have a clear understanding of the essential elements of information relative to the incident. The larger an incident or disaster, the more parties are involved in providing inputs to EEI and receiving EEI outputs, which in turn help generate a shared SA and COP.

As it turned out, before the massive landslide the local responders had few, if any, detailed and coordinated plans in place for a disaster of this kind and magnitude, nor were they prepared for systematically collecting EEI inputs and disseminating EEI outputs to response units and other important stakeholders. Initially, information was rather unsystematically collected and disseminated. A local responder put it this way,

When $T$. < the first incident commander on day 1 - insertion by authors $>$ got there we started setting up some incident joint command with the state patrol and the sheriff's office and fire. We split a few frequencies off the main channel so we could talk separately. So there were some incident command stuff that was happening, but as far as training on what to do out there, it was like kids out in the mud puddle. There was just nothing. Initially we just blindly went out there and tried to find the people...

When the WA State EMD and FEMA Region X support teams along with mutual aid teams from the region among others began to provide organizational assistance, the information situation improved.

Interviewees reported on several barriers and impediments that made it difficult to collect, integrate, and share EEI as quickly as desired:

Technical barriers: The infrastructure for information sharing such as file systems, email distribution lists, logistics systems, and networking arrangements was heterogeneous to an extent, which made it impossible to easily, timely, and comprehensively share documents and messages among responders with a need to know. Furthermore, media breaks in the form of concurrently circulated, but not integrated paper documents, emails, and electronic documents led to an incomplete and distorted operating picture. But even electronic media could not readily be exchanged for the lack of an integrated secure interoperability platform for vertical exchanges among responders of different levels of government and for horizontal exchanges among responders from different agencies. For example, according to one FEMA interviewee,

Our systems have never, at least for as long as I've been here, never been interoperable with other systems. Our WebEOC does not talk to the State's WebEOC and vice versa. And that's the way it's always been. The FEMA firewall's always been an issue, and we know that, so we don't try to solve it because we can't. So we email stuff, basically.

Organizational barriers: While technical barriers contributed their part to the overall difficulties of in- 
formation sharing and coordinated incident action planning (IAP), organizational weaknesses also posed additional barriers in this regard. Standards for inter-responder information sharing and messaging were missing so that completeness and timeliness of information provision was questionable. This was in part due to unclear responsibilities regarding not only what were the respective EEI, but also who would collect them, and with whom these were then to be shared, and when. As one responder observed,

I think the next one was organizational, in that there was never, certainly in the first 48 to 72 hours, I don't think there was ever a clear delegation of authority. So that, I don't know that there was ever clarity of the ... In many ways, the operations was operating at a different level or a different tempo than the oversight and the planning.

While relatively early into the incident response conference calls were held and situation reports (sitreps) were compiled and circulated at various levels on a daily and half-daily basis, in the earlier phase of response despite the availability of better information the sitreps were incomplete due to the lack of sufficient integration of information from available sources.

The aforementioned media breaks along with unrecorded face-to-face communications added to the lack of information integration. No procedure was in place, which would track information flows and information utilization with regard to respective actions taken. Said one local responder,

I was overwhelmed and I didn't even know it. I didn't feel like I was overwhelmed. I didn't feel that world spinning, adrenaline rush out of control. But didn't recognize that I was overwhelmed until days later when I listened to the radio tape. I was like, "Oh my god, I missed some calls. I missed a lot of things" I started to think about some of the things I had written down.

Other barriers, which were both technical and organizational in nature, include the difficulty of scaling information acquisition, dissemination, and utilization processes in an ever more complex response environment, in which finally over 100 government agencies of all levels became involved. Moreover, information access regulations and related protocols made it difficult to share information between teams, for example, between FEMA, State, County, and the IMT. As one responder pointed out,
It's sensitive information, and so there are rules and protocols for how that information can be shared.

This led to some unique arrangements, which another responder described like this,

They worked out ways to transfer that information, which was laborious, physically getting an encrypted hard drive, giving that to the ICP, so they could put their data on that, and then driving to Arlington to get that, and then driving that back.

In summary, technical and organizational barriers played a major role in hampering effective information sharing among responders of various levels. As a result, SA and a shared COP were slowly established, and as a consequence incident action planning was sub-optimally coordinated between responder teams.

5.3 Ad research question $\# 3$ (other challenges including information flow challenges):

As already presented the lack of standards for information sharing and messaging slowed down the flow of information among and between responders. However, other challenges included the mix and operations of synchronous (for example, face-to-face, via cell phone, satellite phone, landline, conference calls, or radio) and asynchronous communication (for example, email or postings).

Synchronous Communication. Radio communication has remained the most important tool for coordinating and messaging among responder teams. This was not any different in the Oso/SR530 landslide response. Initially, however, many teams found themselves with incompatible radios and radio frequencies, which needed updating and patching in order to communicate. Since in the first few days also cell phone connections were weak or spotty, while the fiber cable between the Arlington and Darrington sites was cut by the slide, workarounds had to be implemented in order to enable synchronous communication. FEMA and mutual-aid partners were able to overcome these technical difficulties relatively quickly. However, whereas synchronous communication is indispensable and critically important in disaster response management, important information exchanged in synchronous mode is often not documented and shared with other responders who have a need to know, which might hamper the information flow to and from those responders not directly involved in the synchronous exchange.

Mostly, everybody uses their own personal cell phones. We have found that to be the best way, you know, you're just talking. The prob- 
lem with that, I will say, is, with the three-day turnover, then that's the contact that you had, whoever they were, and then they were gone.

Face-to-face communication was essential; however, the communicated information could easily be lost, whenever staff turned over and the respective information had remained unrecorded. Another responder remarked in this context,

In general, information got shared every couple hours when there were debriefs. It was still pretty chaotic. I'll just speak for me. When there was something specific for me, I would go out and talk to the people in the field do get a better handle on it.

In other words, spoken and written information was insufficiently integrated, or incompletely recorded, so that responders had to double-check before action could be taken.

Asynchronous Communication. Email, texting, and postings were widely and frequently used during the Oso/SR530 landslide response. Also, print documents were circulated. The known crux with asynchronous communication is (a) the missing acknowledgement from the receiving side and (b) the undetermined consequence and action resulting from the input. Cases were reported that requests and important information shared via email or postings had remained unattended, and no action was taken demonstrating the weakness of this particular way of communication in mostly time-critical disaster management. One responder was very clear about this,

Your email is not a form of communication, it's actually a way to share some information, but if you actually need to communicate with somebody, you may send them an email, and then you should pick up the phone and say 'Hey, I know this sounds odd, but I sent you this email, it's got some information in it, I need you to look at it and we can either talk while you're looking at it, or you need to call me back.

Geographical Obstacles. While the Western (Arlington) side of the landslide was easily accessible for arriving response teams, the Eastern (Darrington) side was literally cut off when the State Route 530 had been covered by a thick and wide layer of debris, which for months would effectively isolate the uppervalley communities from easy access to supplies and services. Interestingly, also in the management of at least the initial response the Darrington side was not as integrated as the Arlington side, which became visible, for example, in an imbalance of resource al- locations favoring the Western side. The Darrington side also had a relatively weak information infrastructure with slow network connections making it difficult for the incident command post located in Arlington and the County EOC in Everett to communicate with the Eastern side. As a result, information flows between the three sides were relatively slow, and IAPs were initially relatively loosely coordinated. One interviewee remembered,

There was very limited reporting. There were only two situation reports, I think, going out on a day, and they changed very little. When I developed a written donations plan and status, and had included a list of the contacts, I asked them where I should house it. They said, "Just keep it." It's like, well, that doesn't work. You know, nobody knows about it. There was no place to put it. So I emailed it to the planning unit, but they did nothing with it, to my knowledge. And I emailed it out to a bunch of other people, too. Same thing with reunification for personal belongings.

Similarly, the USAR team operating on the Eastern side of the debris field also complained about a lack of planning and cooperation on part of the County (in terms of mass fatality handling) and a lack of coordination on part of the IMT at Arlington, which led the USAR team to skipping protocol and making their own decentralized decisions and IAPs. Said one response leader,

In this incident, the medical examiner never showed up, so all investigation was handled by the task force, all documentation was handled by the task force, and we did a great job. I'm very proud of the job that was done. But that's not what we normally do. Normally we find them, extract them, you know, to a place that ... even before we extract them there's somebody documenting it. But in this case, there was none of that. We handled it, and then, in addition, we handled all the protocol for how were we going to move them in the presence of family, in the presence of relatives, and there were no policies that were established by command.

Local Community Involvement. Despite grief and shock, local residents and family members were important sources of information guiding the search and rescue operations. Local community members were the first to accurately inform the arriving response teams of the potential damage to life and property, which prompted the ramping up of resources and provided valuable information to the response teams 
on-site. The locals were also of important help when locations of missing landmarks needed identification, and when the best access points to the debris field had to be determined. On the other hand, by involving family and community members in the response, the responders provided a tremendous opportunity for managing the community and families' difficult emotional situation. When approached by enraged and highly concerned family members, responders calmed the situation by sharing their plan and offering a way to involve them as volunteers. As one responder reported saying,

This is our plan... If somebody is out there, then we are going to find them, but this is the procedure. You can either be part of it, or you can go away, and if you don't go away, we can have the sheriff whisk you away. I don't want to push you away, I want you to be involved, and here is the way you can be involved, but you have got to cooperate. It totally changed their demeanor. We never had any issues here. It was pretty amazing.

Working with the urban search and rescue teams the volunteers had an opportunity to serve as effective sources of information.

In summary, other challenges to gaining and maintaining SA and a shared COP leading to coordinated IAPs included the modes of information exchange (synchronous and asynchronous) along with geographical obstacles. Involving locals, in contrast, significantly helped responders on site understand the situation and navigating the debris field.

\section{Discussion}

Situation Awareness According to the Endsley Framework. The State Commission Report of December 2014 [20] claims that the "magnitude of the SR530 Landslide was not fully comprehended for several hours <emphasis by authors $>$ " (p. 8). This representation, however, is incorrect; it should rather read "for several days." When using the aforementioned Endsley framework of situational awareness $[9,11]$ in this case, it is evident that basic level-1 SA (knowing the basic facts) was reached no sooner than day 4), whereas level-2 SA (comprehending the situation) emerged around the time the first type-2 team was brought in on day 6, and level-3 SA (projecting and anticipating future developments) took yet another few days, which became very clear through the interviews, but which is also welldocumented and detailed in the FEMA Daily Operations Briefings in the first couple of weeks (see htwww.disastercenter.com/FEMA+Daily+Ops+Briefi
ng+INSERT_DATE.pdf) after the landslide. The incorrect representation of SA/COP-related facts, however, unfortunately, leads to overlooking the seriousness of systemic problems unveiled in this study.

Ex-ante Planning for Collecting EEI. Given the duration, scale and scope of the incident, one can classify the Oso/SR530 landslide "massive" for a small town, or "partial" for a small city, which would make it a category-4 to -5 disaster according to Fischer's ten disaster categories [13]. It is noteworthy that a disaster of a medium category can already be highly demanding to local, State, and federal response efforts, not least of which in terms of shared SA, shared COP, and coordinated IAPs. Snohomish County and its municipalities were caught by this incident obviously flat-footed.

While the County Emergency Management Department with a FTE staff of fourteen had some plans in place, it had no plans prepared for dealing with massive landslides, and no hazard-specific EEI had been developed, which would have helped coordinate the various response teams and efforts more easily. As the disaster response revealed, the County also did not have a pre-coordinated plan for dealing with mass fatalities. Also, the County EOC was not even fully activated during the incident response and left without leadership, since the EMD director decided to involve himself at the ICP in Arlington, while simultaneously the Deputy EMD Director was deployed to the Darrington side, which introduced additional confusion, coordination problems between IMT and EOC, and added to the lack of both SA and COPbased coordination.

Furthermore, authority for carrying out response efforts by other and high-capability partners appeared to have been delegated in fairly limited and sort of ambiguous ways. This had a direct detrimental influence on almost all aspects related to shared SA, shared COP, and shared IAPs.

In other words, the level of preparedness on part of the County for a disaster of this extent appears to have room for improvement including the development of a sound understanding and practice of EMD leadership roles during an unfolding disaster.

Missing Standards for Information Sharing. In the United States, incidents are regularly managed on the basis of structures and standards defined in the $\mathrm{Na}$ tional Incident Management System (NIMS) and the Incident Command System (ICS) at its core. ICS employs a hierarchical command-and-control structure with clear responsibilities along with interfaces between various general staff sections (operations, planning, logistics, and finance/administration) and the command staff (public information, safety, and 
liaison). Incident management teams (IMTs) under ICS work with local emergency operations centers (EOCs), which coordinate resources and incident support along fifteen emergency support functions (ESFs). While the IMT under ICS is hierarchically organized [5], which has been much criticized in the academic literature for various reasons $[2,7,28]$, the EOCs on various levels provide essential capabilities and resources, which make the whole response effort function more like a hybrid or matrix organization rather than a pure and simple hierarchical organization.

In an emergent complex organization like the Oso/SR530 landslide response, which had numerous ad-hoc elements and entities requiring coordination, effective vertical and lateral flows of information are of the essence. However, standards or unified protocols for information sharing and messaging appear to be missing. Such content-related standards would, for example, include but are not limited to the aforementioned hazard-specific templates for EEI, lists of EEIrelated information owners/providers, distribution lists of responders and response support functions with a need to know, and information distribution tracking mechanisms.

Lack of Information Integration. In a complex multi-agency incident response like the Oso/SR530 landslide response, information is collected, generated, and shared in various modes and formats. Information integration was missing due to various factors, for example, such as media breaks, that is, pieces of information were recorded and shared on paper, others were maintained electronically, and yet others were communicated verbally; however, the various pieces were not systematically put together, which led to ambiguity and a distorted COP. Other obstacles hampering information integration included unclear information dissemination procedures and frequent staff turnovers, which led to losses of important information.

Lack of information sharing and integration results in a phenomenon known as "siloing" [1]. Information silos emerge, whenever information is retained by individuals or not widely shared for a variety of different reasons mentioned above. Evidence of siloing manifested in the interviews when respondents complained that information they needed existed, but was not accessible. It has to be noted that information silos would not automatically be dissolved with the introduction of more advanced technology. Instead management practices and information architecture issues must be addressed to resolve siloing.
In order to improve inter-unit information sharing and coordination, various response units deployed liaisons at other units who effectively helped with bidirectional information sharing and coordination. However, the liaisons were able to only connect and report bi-directionally what they noticed in their given roles potentially missing important pieces of information. In consequence, this important area of information integration needs further study to be better understood.

Lack of a Unified Information Architecture and System Platform. Response units used different systems such as SharePoint, WebEOC, Google Docs, simple email, and traditional paper documents to record and organize their tasks and resource requests. The IMT/ICP and the County EOC physically exchanged information once a day via an encrypted hard disk, which was shipped back and forth between the two sites. Moreover, while the County EOC used SharePoint for sharing and storing documents, the State EMD and FEMA region $\mathrm{X}$ utilized their respective versions of Intermedia's WebEOC for task and resource tracking. However, even between the FEMA WebEOC and the State WebEOC data exchanges were made impossible, since the systems were firewalled and shielded against access from and exchanges with the outside world. In contrast, the type2 IMT employed a low-tech approach using email, paper documents, and the aforementioned encrypted hard disk for information sharing, all of which would not make information sharing timely and easy. A similar multitude of approaches and platforms was found also in the area of resource requesting, on which the project team reports in a separate paper on "managerial challenges." In a nutshell, information sharing and information processing among and between response units was rather cumbersome and slow during the response, at least in the earlier stages.

As pointed out, the larger and the more dynamic an incident the larger the ad-hoc matrix organization handling the incident inevitably grows. While basic interfaces underneath the NIMS/ICS and EOC/ESF umbrellas are relatively well defined, it is surprising that the most important enabler and potential facilitator of a complex matrix organization in an unfolding incident, that is, a unified information architecture and a corresponding information system platform has remained undefined and non-standardized.

What might work well for a single-jurisdiction EOC and a single IMT/ICP when responding to a smaller scale/scope/duration incident, provided they use the same information architecture and system platform (for example, with WebEOC), appears to not scale well at all, once an incident of larger scale, 
scope, and duration is at hand. In the aftermath of the Oso/SR530 landslide, the State of Washington standardized its emergency-related resource request and tracking procedures, also introducing standardized forms. It appears that a similar approach to define and devise a unified system architecture by standardizing secure information processing, information organization and record management, information sharing procedures, information system platforms, and application program interfaces (APIs). As suggested in the previous section, more research is needed also in this particular area.

The Commission Report. In light of the findings of this study, the State Commission Report [20] correctly points at major problems with gaining and maintaining situational awareness, and it distinguishes four categories of infrastructure, interoperability, content, and strategy, into which these "issues" fall (p. 28). However, when analyzing the causes, the report mostly refers to problems and failures in the physical and technological infrastructures and their consequences for system interoperability. Hence it misses to identify problems rooted much more deeply and more widely, which this study unveiled. As a result, the Report downplays the severity of the overall problem and simply concludes to recommend the implementation of better and more technology such as the proposed Federal initiative of FirstNet (www.firstnet.gov/) and the use of drones for better reconnaissance. While undoubtedly highspeed wireless digital data connections could greatly improve information exchanges in disaster response, the aforementioned deeper problems of lacking exante planning of EEI, lacking information integration, and lacking a unified Information Architecture and System Platform would still remain unaddressed. Furthermore, using drones in a tight airspace while simultaneously airborne operations were carried out by helicopter search and rescue teams would have introduced great safety risks and uncontrollable complexity for air control, which is why air operations commanders turned down respective requests. However, immediate higher-altitude reconnaissance flights, for example, by fixed-wing aircraft equipped with high-resolution photographic and forwardlooking infrared radiometer (FLIR) capabilities might have given a more comprehensive picture of the impacted area sooner.

In summary, problems of gaining and maintaining shared SA and arriving at a shared COP leading to coordinated IAPs encompass intertwined problems related to planning, information sharing, information integration, information architecture, and information system platforms, which need further study. More advanced technology alone will not resolve the identified problems.

\section{Conclusions}

It has been the object of this study to explore and identify major problem areas of information management in disaster response, which hinder the rapidly development and maintenance of shared situational awareness and a shared common operating picture. Responders' diverse information needs, their information behavior throughout various stages of responding, and the respective information flows have not been systematically identified.

In the course of the study, it was found that lack in hazard-related planning hampered systematic information collection and sharing; also, the lack of standardized information sharing procedures and information integration practices were found detrimental to gaining and maintaining shared SA/COP. Furthermore, the absence of a unified information architecture and information system platform further exacerbates the information "siloing" problem. This study strongly suggests that further study in these particular areas is necessary. In parallel, the project team also studied related "managerial challenges" in the Oso/SR530 landslide response, which are published in a separate paper.

This study reports on the response in the single case of the 2014 Oso/SR530 landslide. A study of a single case inherently introduces certain limitations with regard to the transferability of results. However, while this particular disaster ranks in the medium section of the Fischer Disaster Scale [13], the observed complexities in the response, nevertheless, provide ample evidence of SA/COP-related problems to be systemic.

\section{Acknowledgement}

The following research assistants participated in the transcribing, coding, concept analyzing and mapping of the interviews: Stephanie E Ballard, Jorge Borunda, Ammi Bui, Tim Carlson, Sarah Carnes, Sarah Carrier, Yi Fan Chang, Tiffany B Coulson, Amanda Cummings, Heather Diaz, Sherry Shiyu Gao, Andy Herman, Akashdeep Singh Jaswal, Tanu Khandelwal, Cassondra Koldewyn, Divya Kothari, Deborah Kyle, Josephine Lau, Kung Jin Lee, Lysia Li, Audrey Lorberfeld, Elan May Rinck, Randi L Mendel, Grace Morris, Neal Parker, Liz Pritchard, Deepa Sudarshan Rao, Dan Ray, Jorge Retamales, Tricia M Rhodes, Rashmi Sharma, Yuzhou Shen, Gagandeep Singh, Leili E Slutz, Louis C Spinelli, Sonal Srivastava, Jenna Telvik, Emily Thompson, Aubrey Walter, Vaibhav Walvekar, Grant Woods, Fan Yang, April Ybarra, Katiana D Yeo, Yunjie Zhou, Yao Zhou, and several graduate assistants from Graduate Assistant Team. Special thanks to Sue Morgan who organized and orchestrated the online streaming and video recording of the weekly project sessions. 


\section{References}

[1] Beachwood, O. H., F. L. Weston, C. A. Coronado, F. L. Hollywood, and M. C. M. E. Calendar, "Electronic siloing: An unintended consequence of the electronic health record," Cleveland Clinic Journal of Medicine, vol. 80, pp. 406-409, 2013.

[2] Buck, D. A., J. E. Trainor, and B. E. Aguirre, "A critical evaluation of the incident command system and NIMS," Journal of Homeland Security and Emergency Management, vol. 3, 2006.

[3] Case, D. O., Looking for information : a survey of research on information seeking, needs, and behavior. Amsterdam ; Boston: Elsevier/Academic Press, 2007.

[4] Chua, A. Y. K., S. Kaynak, and S. S. B. Foo, "An Analysis of the Delayed Response to Hurricane Katrina Through the Lens of Knowledge Management," Journal Of The American Society For Information Science And Technology, vol. 58, pp. 391-403, 2007.

[5] Cole, D., "The incident command system: A 25-year evaluation by California practitioners," ed: National Fire Academy, 2000.

[6] Coyle, D. and P. Meier, "New technologies in emergencies and conflicts: The role of information and social networks," United Nations FoundationVodafone Foundation Partnership, Washington, DC and London, UK2009.

[7] Drabek, T. E. and D. A. McEntire, "Emergent phenomena and multiorganizational coordination in disasters: Lessons from the research literature," International Journal of Mass Emergencies and Disasters, vol. 20, pp. 197-224, 2002.

[8] Endsley, M. R., "Measurement of situation awareness in dynamic systems," Human Factors: The Journal of the Human Factors and Ergonomics Society, vol. 37, pp. 65-84, 1995.

[9] Endsley, M. R., "Toward a theory of situation awareness in dynamic systems," Human Factors: The Journal of the Human Factors and Ergonomics Society, vol. 37, pp. 32-64, 1995.

[10] Endsley, M. R., "Situation awareness misconceptions and misunderstandings," Journal of Cognitive Engineering and Decision Making, vol. 9, pp. 4-32, 2015.

[11] Endsley, M. R., "Situation awareness: operationally necessary and scientifically grounded," Cognition, Technology \& Work, vol. 17, pp. 163-167, 2015// 2015.

[12] Fereday, J. and E. Muir-Cochrane, "Demonstrating Rigor Using Thematic Analysis: A Hybrid Approach of Inductive and Deductive Coding and Theme Development," International journal of qualitative methods, vol. 5, 2006.

[13] Fischer, H. W., "The Sociology of Disaster: Definitions, Research Questions, \& Measurements Continuation of the Discussion in a Post-September 11 Environment," International Journal of Mass Emergencies and Disasters, vol. 21, pp. 91-107, 2003.
[14] Glaser, B. G., "The Future of Grounded Theory," Qualitative Health Research, vol. 9, pp. 836-845, November 1, 19991999.

[15] Glaser, B. G. and A. L. Strauss, The discovery of grounded theory; strategies for qualitative research. Chicago,: Aldine Pub. Co., 1967.

[16] Harrald, J. R., "Agility and Discipline: Critical Success Factors for Disaster Response," The ANNALS of the American Academy of Political and Social Science, vol. 604, pp. 256-272 2006.

[17] Harrald, J. R. and T. Jefferson, "Shared situational awareness in emergency management mitigation and response," in 40th Annual Hawaii International Conference on System Sciences (HICSS-40), Waikoloa, HI, 2007, pp. 23-30.

[18] Kapucu, N., "Interagency Communication Networks During Emergencies. Boundary Spanners in Multiagency Coordination," American Review of Public Administration, vol. 36, 2006.

[19] Kapucu, N., T. Arslan, and M. L. Collins, "Examining intergovernmental and interorganizational response to catastrophic disasters: Toward a network-centered approach," Administration \& Society, vol. 42, pp. 222247, 2010.

[20] Lombardo, K., J. Boggs, J. Boudreau, P. Chiles, J. Erickson, W. Gerstel, et al., "SR 530 Landslide Commission Final Report," S. L. Commission, Ed., ed. Olympia, WA: SR 530 Landslide Commission, 2014, p. v/49.

[21] Mendonça, D., T. Jefferson, and J. Harrald, "Collaborative adhocracies and mix-and-match technologies in emergency management," Communications of the ACM, vol. 50, pp. 44-49, 2007.

[22] Palen, L., S. Vieweg, and K. M. Anderson, "Supporting "Everyday Analysts" in Safety- and Time-Critical Situations," The Information Society, vol. 27, pp. 52-62, 2011.

[23] Ritchie, J., J. Lewis, and E. Gillian, "Designing and selecting samples," in Qualitative research practice : a guide for social science students and researchers, J. Ritchie and J. Lewis, Eds., ed London ; Thousand Oaks, Calif:: Sage Publications, 2003, pp. 77-108.

[24] Scholl, H. J. and B. J. Patin, "Resilient Information Infrastructures: Criticality and Role in Responding to Catastrophic Incidents," Transforming Government: People, Process and Policy, vol. 8, pp. 28-48, 2014.

[25] Strauss, A. L. and J. M. Corbin, Basics of qualitative research : techniques and procedures for developing grounded theory, 2nd ed. Thousand Oaks: Sage Publications, 1998.

[26] Turoff, M., "Emergency response information systems: Emerging trends and technologies," Communications of the ACM, vol. 50, pp. 29-31, 2007.

[27] Urquhart, C., H. Lehmann, and M. D. Myers, "Putting the 'theory' back into grounded theory: guidelines for grounded theory studies in information systems," Information Systems, vol. 20, pp. 357-381, 2010.

[28] Wenger, D., E. L. Quarantelli, and R. R. Dynes, "Is the Incident Command System a Plan for all Seasons and Emergency Situations?," Hazard Monthly, vol. 10, pp. 8-12, 1990. 